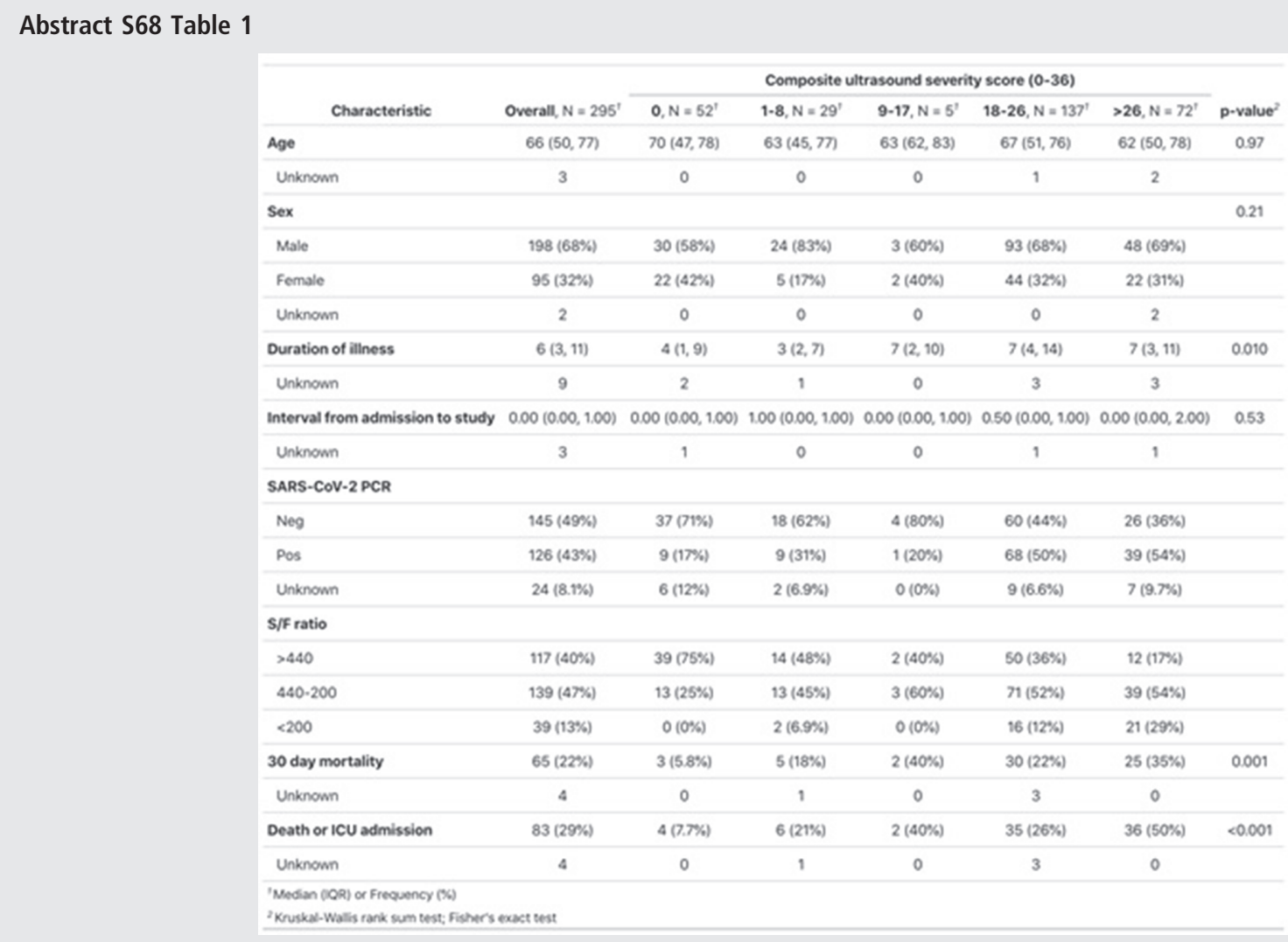

significant in a multivariate model controlled for age, sex, the time interval from admission to scan and the severity of hypoxia.

Conclusions LUS discriminates between patients at increased risk of deterioration. The ultrasound severity score appears to be best calibrated with risk in patients receiving oxygen therapy. The evaluation provides further evidence of the clinical utility of LUS which combined with the potential practical advantages provide a strong argument for wider adoption and integration of the practice.

\section{S69 INFLAMMATORY BIOMARKERS PREDICT CLINICAL OUTCOMES IN PATIENTS WITH COVID-19 INFECTION: RESULTS FROM THE PREDICT-COVID19 STUDY}

${ }^{1} \mathrm{MB}$ Long, ${ }^{1} \mathrm{HR}$ Keir, ${ }^{1} \mathrm{YH}$ Giam, ${ }^{1} \mathrm{H}$ Abo Leyah, 'T Pembridge, 'L Delgado, ${ }^{2} \mathrm{R}$ Hull, ${ }^{1}$ A Gilmour, ${ }^{1} \mathrm{C}$ Hughes, ${ }^{1} \mathrm{C}$ Hocking, 'BJM New, 'D Connell, ${ }^{1} \mathrm{H}$ Richardson, 'DM Cassidy, ${ }^{1}$ A Shoemark, 'JD Chalmers. 'University of Dundee, Dundee, UK; ${ }^{2}$ University of Sheffield, Sheffield, UK

\subsection{6/thorax-2021-BTSabstracts.75}

Introduction COVID-19 is reported to cause profound systemic inflammation. Anti-inflammatory treatments such as corticosteroids and anti-IL-6 receptor monoclonal antibodies reduce mortality. Identifying inflammatory biomarkers associated with increased morbidity and mortality may allow both prediction of outcomes and identification of further therapeutic targets.

Methods A prospective observational study of patients with PCR-confirmed SARS-CoV-2 admitted to a single centre in Dundee, UK. Patients were enrolled within 96 hours of hospital admission. 45 inflammatory biomarkers were measured in serum using the Olink Target48 proteomic-based biomarker panel. Additional markers were measured by ELISA/ immunoassay and enzyme activity assays. Severe disease was defined as the requirement for non-invasive or mechanical ventilation or death within 28 days of admission. Discrimination between groups was evaluated using the area under the receiver operator characteristic curve (AUC).

Results 176 patients were included (mean age 64.9 years, SD 13.6), 101 were male (57.4\%). 56 patients developed severe disease (31.8\%), mortality was $16.5 \%$. Using ROC analysis, the strongest predictors of severity $(\mathrm{p}<0.0001)$ were CCL7/MCP3 (AUC 0.78 95\%CI 0.70-0.85), IL6 (0.73 95\%CI $\quad 0.66-0.81), \quad$ IL15 $\quad(0.73 \quad 95 \%$ CI $\quad 0.65-0.81)$, CXCL10/IP10 (0.73 95\%CI 0.65-0.81). Further significant predictors of severity included CXCL11, IL10, CCL2/MCP1 and CSF2/GM-CSF. Predictors of mortality were CXCL10 (0.78 95\%CI 0.69-0.86), IL6 (0.76 95\%CI 0.67-0.85), IL15 (0.75 95\%CI $0.66-0.84)$, IL10 (0.73 95\%CI $0.64-$ 0.82). Further significant predictors of mortality were CXCL9 and CCL7.

Conclusion Multiple circulating biomarkers were identified which predicted disease severity and mortality in COVID19, indicating clinical value in measurement upon hospital admission to highlight high-risk patients. Associated biological processes for these proteins included anti-viral and interferon responses and immune cell chemotaxis. In particular, CCL7 and CXCL10, the strongest predictors of severity and mortality in this dataset, are key players in the cytokine storm and immune cell recruitment linked with COVID19. These chemokines are not currently therapeutic targets, highlighting key avenues for further clinical research. 